\title{
Setor de Atendimento Médico Unificado: criação, finalidades, contribuição para a vigilância e perspectivas
}

\author{
Unified Medical Care Sector: creation, purposes, contribution to health surveillance, \\ and perspectives
}

Ana Yecê das Neves Pinto, Haroldo José de Matos, Francisco Lúzio de Paula Ramos

Instituto Evandro Chagas/SVS/MS, Serviço de Epidemiologia, Setor de Atendimento Médico Unificado, Ananindeua, Pará, Brasil

\begin{abstract}
RESUMO
Estamos presenciando uma complexa transição epidemiológica com a emergência de doenças infecciosas até então desconhecidas e a reemergência de doenças tidas como controladas, sendo o envelhecimento e as mudanças climáticas fatores importantes de vulnerabilidade. O Instituto Evandro Chagas, visando fortalecer sua missão de vigilância em saúde, criou o Setor de Atendimento Médico Unificado a partir de uma demanda de pacientes para esclarecimento diagnóstico de síndrome infecciosa com ênfase em febre prolongada. No decurso do seu nono ano de atuação, o Setor contabiliza 34.057 atendimentos, com média anual de 3.700 pacientes. Dessa demanda, extraem-se informações valiosas para a vigilância epidemiológica e para o fortalecimento do conhecimento sobre doenças emergentes. A febre tifoide e a doença de Chagas, duas das principais causas de febre na região, são exemplos marcantes. Nos últimos cinco anos, foram registrados 308 casos de febre tifoide e a média anual de 15 surtos microepidêmicos de doença de Chagas. A identificação dos agravos tem desencadeado investigações em parceria com as Secretarias Estadual e Municipais de Saúde para o diagnóstico rápido, oportunizando também a geração de evidências científicas potencialmente aplicáveis na atenção e na prevenção. Assim, a criação do serviço de atenção médico-diagnóstica em uma instituição de pesquisa médica visou não apenas fortalecer a pesquisa gerando conhecimento, mas, sobretudo, fortalecer a estratégia de diagnóstico rápido em endemias regionais, além de implementar a pesquisa de aplicação imediata de resultados a populações negligenciadas em sintonia com a resiliência própria do compromisso institucional com a saúde pública.
\end{abstract}

Palavras-chave: Vigilância Epidemiológica; Surtos de Doenças; Febre Tifoide; Doença de Chagas; Pesquisa Médica Translacional.

\begin{abstract}
We are witnessing a complex epidemiological transition with the emergence of previously unknown infectious diseases, and the re-emergence of diseases that were believed to be under control, with aging and climate change as important factors of vulnerability. The Instituto Evandro Chagas created the Unified Medical Care Sector (Setor de Atendimento Médico Unificado) to strengthen its health surveillance mission. This was a result of patients demand for diagnostic clarification of infectious diseases with an emphasis on prolonged fever. During the ninth year of its creation, the Sector has had 34,057 hospital visits and an annual average of 3,700 patients. From this data, valuable information was extracted for epidemiological surveillance and for strengthening understanding about emerging diseases. The data for typhoid fever and Chagas disease, two leading causes of fever in the region, were striking. In the previous five years, 308 cases of typhoid fever was recorded and there was an annual average of 15 micro-epidemic outbreaks of Chagas disease. The identification of diseases trigger the investigations in partnership with the State and Municipal Health Secretariats for quick diagnosis. Moreover, it supports the generation of scientific evidence potentially applicable to health attention and prevention. Thus, the creation of the medical-diagnostic attention service in a medical research institution has not only strengthened the researches that generate knowledge but also the rapid diagnostic response to regional endemic diseases. Furthermore, it has enabled the researches on immediate application of results to relatively neglected populations, which is in line with the institutional commitment to public health.
\end{abstract}

Keywords: Epidemiological Surveillance; Disease Outbreaks; Typhoid Fever; Chagas Disease; Translational Medical Research.

\footnotetext{
Correspondência / Correspondence:

Francisco Lúzio de Paula Ramos

Instituto Evandro Chagas, Serviço de Epidemiologia, Setor de Atendimento Médico Unificado

Rodovia BR-316 km 7, s/n. Bairro: Levilândia - CEP: 67030-000 - Ananindeva, Pará, Brasil

E-mail: franciscoluzio@iec.pa.gov.br
} 


\section{INTRODUÇÃO}

Estamos vivendo uma nova transição na saúde pública em escala mundial, poderíamos denominá-la transição para a complexidade. Classicamente, a transição epidemiológica traduz a passagem da era do predomínio da mortalidade proporcional por doenças infecciosas e parasitárias para outra realidade na qual a mortalidade proporcional por doenças crônicas degenerativas passaria a predominar. Vários fatores atuariam nesse contexto, sendo que a transição demográfica, com o aumento progressivo do envelhecimento da população, seria um dos mais fortes determinantes.

No entanto, essa transição não vem ocorrendo da forma como inicialmente fora prevista no estudo clássico de Omran de 1971'. Ao contrário, conforme se observa nas quatro últimas décadas, defrontamo-nos com desafios nunca imaginados nos anos de 1960 e 1970 na área das doenças infecciosas. É a realidade das doenças infecciosas emergentes e reemergentes, que se choca com a realidade prevalecente das doenças crônicas degenerativas.

No Brasil, e muito especialmente na Amazônia brasileira, essa complexidade adquire um tom de contornos ainda mais marcantes e imprevisíveis, pela presença de condições ecoepidemiológicas que favorece, há muitas décadas, a permanência de um conjunto de doenças endêmicas, como a malária, doença de Chagas (DC), tuberculose, hanseníase e febre tifoide (FT) entre outros agravos ${ }^{2}$. Por outro lado, o desafio das chamadas doenças crônicas degenerativas não se reduz somente ao grande incremento da mortalidade por doenças cardiovasculares ou por câncer; mas um desafio rapidamente progressivo está expresso pelo grande aumento dos óbitos por causas externas, notadamente por acidentes de trânsito e pela violência urbana, particularmente entre a população jovem ${ }^{3}$.

Essa realidade complexa de transições, sem mencionar a transição climática que o planeta experimenta, e que, na Região Norte, se expressa em cheias históricas dos seus numerosos rios, vem se tornando um desafio constante para os gestores e profissionais da saúde pública.

Esse desafio requer repetidas intervenções na área da vigilância epidemiológica e da formação em Epidemiologia, pois muitas vezes o profissional de saúde, envolvido na gestão ou na execução das múltiplas tarefas da saúde pública, não teve a formação orientada para a realidade multifacetada da saúde pública atual na nossa região.

Uma das tarefas básicas da vigilância epidemiológica é a notificação dos agravos e doenças de notificação compulsória. Esse conjunto reúne agravos e doenças que podem ser classificados como de interesse local, regional, nacional ou internacional, em função de seu impacto sobre as populações e do risco potencial de epidemias. Nesse sentido, essa lista é bastante dinâmica e é revista periodicamente. A última atualização encontra-se na Portaria $n^{\circ} 204$ de 17 de fevereiro de 2016 do Ministério da Saúde (MS), e foi realizada em função da inclusão da notificação da doença pelo vírus Zika, a mais recente emergência epidemiológica entre nós ${ }^{4}$.

Na estrutura da vigilância epidemiológica brasileira, as fichas das doenças de notificação compulsória compõem uma base de dados nacional constituída no Sistema de Informações de Agravos de Notificação (SINAN). Esse sistema tem a gestão direta da Secretaria de Vigilância em Saúde (SVS) do MS, à qual o Instituto Evandro Chagas (IEC) está ligado. Há também uma gestão estadual, por meio das Secretarias Estaduais de Saúde, e uma gestão municipal, por meio das vigilâncias epidemiológicas das Secretarias Municipais de Saúde.

Em janeiro de 2013, o Serviço de Epidemiologia do IEC (SEVEP/IEC) tornou-se uma unidade notificadora do SINAN por meio da instalação de uma versão atual desse sistema em um computador local, propiciando a notificação dos agravos detectados no Setor de Atendimento Médico Unificado (SOAMU), num processo de transferência eletrônica do banco de dados para a Secretaria Municipal de Saúde de Ananindeua, uma vez que o IEC está localizado nesse Município. Isso representou um grande impacto na vigilância epidemiológica não só para o Município de Ananindeva, mas para todo o Estado do Pará, pois ao IEC, historicamente, são referenciados pacientes de vários municípios paraenses e até de outras Unidades da Federação, como também de fora do País. Atualmente, os dados da base do SINAN/IEC tornam-se públicos pelo Boletim Epidemiológico do SEVEP/IEC $5,6,7$.

Outra grande contribuição do SINAN é a oportunidade de identificar precocemente as urgências epidemiológicas. Nessa perspectiva, desde a sua criação, - IEC tem a tradição de contribuir para a investigação de surtos e epidemias em nossa região desde a sua criação. A Instituição também tem colaborado, nessa linha de ação, com outras instituições, como as Secretarias Municipais, a Secretaria Estadual de Saúde do Pará (SESPA) e até mesmo a articulação em nível federal, em particular com o Programa Epidemiologia Aplicada aos Serviços do Sistema Único de Saúde (EpiSUS) da SVS/MS.

Ao longo do processo da estruturação de um setor apto a executar atividades de vigilância epidemiológica, vem surgindo a necessidade de adequá-lo também às demandas ativas de pessoas que buscam elucidação diagnóstica rápida para suspeitas de doenças endêmicas. Sendo algumas dessas doenças de caráter desconhecido entre nós (emergentes), tornou-se fácil integrar as novas demandas de vigilância epidemiológica à secular tradição institucional para a pesquisa biomédica.

\section{O IEC E A ATENÇÃO DIAGNÓSTICA À DEMANDA DE PESSOAS: SURGIMENTO E FINALIDADES DO SOAMU}

IEC foi criado para a pesquisa, tanto que o seu primeiro nome foi Instituto de Pathologia Experimental 
do Norte (Ipen), dirigido por seu fundador, o dr. Evandro Serafim Lobo Chagas 8 . O objetivo maior do seu grande idealizador era estudar o calazar, uma doença nova que fora vista, à época, nas Regiões Norte e Nordeste. No entanto, não demorou para o dr. Evandro Chagas perceber que não tinha como separar a pesquisa da vigilância em saúde. Era imprescindível registrar aquela e outras doenças para propiciar seus controles. E O IEC seguiu, desde então, sua brilhante caminhada voltado para estas duas vertentes: pesquisa aplicada à saúde, sobretudo de populações amazônidas, e à vigilância em saúde.

Historicamente, aproveitando a expertise do seu corpo técnico e da capacidade de seu aparato laboratorial, as redes pública e privada, reconhecendo sua grande competência, sempre encaminharam ao IEC pacientes portadores de síndromes infecciosas de maior complexidade resolutiva, assim como de doenças consideradas exóticas, para avaliação e auxílio à resolução diagnóstica.

A construção da nova sede do IEC, no Município de Ananindeua, coincidiu com 0 novo panorama institucional, que tomou dimensões tecnológicas compatíveis com o tamanho de sua estrutura física e com a sua capacidade humana. Com isso, era óbvio também o crescimento da demanda de pacientes encaminhados.

Em auxílio à vigilância em saúde, veio a necessidade de se organizar o atendimento voltado a essa demanda de síndrome infecciosa de alta complexidade diagnóstica, mormente a síndrome febril prolongada de origem obscura.

Assim, sob o prisma da vigilância, o SEVEP/IEC, estrategicamente, organizou um setor para centralizar o atendimento médico diagnóstico, vislumbrando esse grande potencial de apoio diagnóstico e a vigilância, suportado por dois pilares: a especificidade dessa demanda, da qual se extrai rica contribuição ao SINAN e o ímpar aparato laboratorial com enorme potencial de resolução. Dessa maneira, logrou-se obter o SOAMU.

Esse setor teve suas atividades iniciadas no ano de 2008. A cada ano, a demanda vem se mantendo em patamares altos, considerando a proposta de atendimento a pessoas suspeitas de doenças de etiologia infecciosa, com ênfase às síndromes exantemática, ictérica e febril prolongada ou, como se convencionou anteriormente denominá-la, febre de origem obscura.

O SOAMU recebe pacientes suspeitos oriundos de todo o Estado do Pará, bem como de outras Unidades da Federação, sobretudo da Região Amazônica, executando trabalhos concatenados às seções técnicocientíficas do IEC. Assim, proporciona qualidade na atenção diagnóstica em doenças infectoparasitárias, utilizando a simples tríade metodológica: suspeição médica precisa - que resulta na imediata notificação; procedimento laboratorial de excelência; e interpretação clínica de resultados imediata repassada ao paciente como intervenção, em consonância com os preceitos do SUS.
No exercício do seu papel voltado à vigilância, - SOAMU tem dado extraordinária contribuição à vigilância epidemiológica do Estado. Nas situações de urgência epidemiológica, esse Setor não só cumpre seu papel de notificar imediatamente o fato, como tem participado da investigação requerida em ação conjunta com as secretarias de saúde envolvidas.

No dia 30 de abril de 2016, assinalou-se o atendimento número 34.057 no SOAMU, cuja média anual tem sido de 3.700 atendimentos (Figura 1).

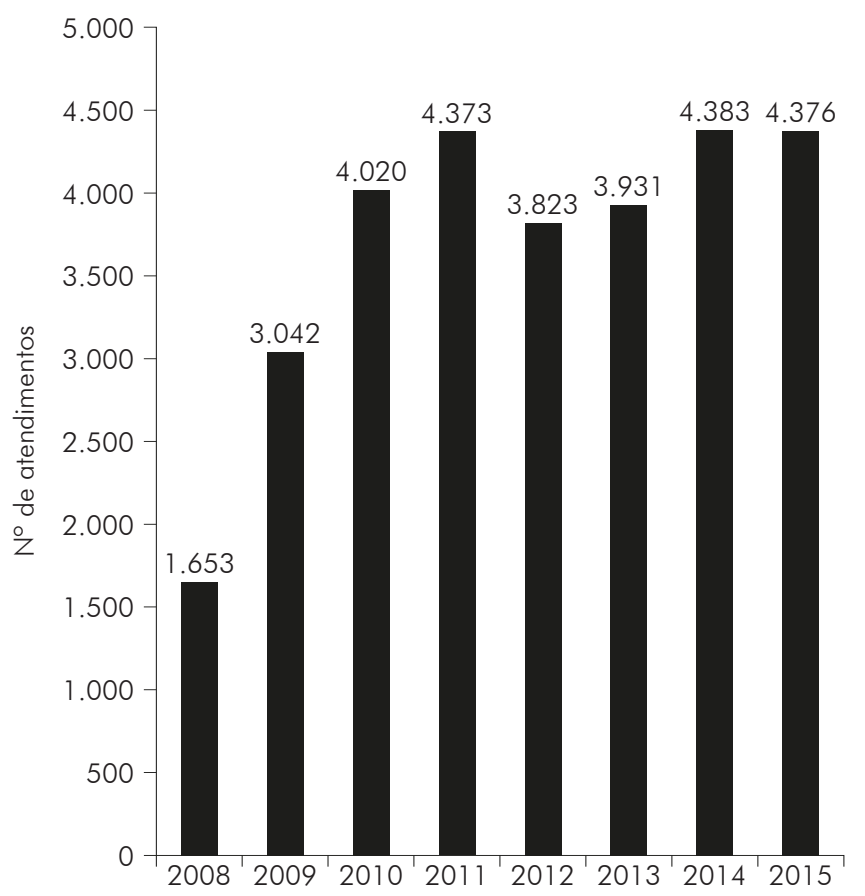

Fonte: Boletim Epidemiológico, ano I, $n^{\circ} 1$, janeiro-abril de 2015; Boletim Epidemiológico, ano I, $\mathrm{n}^{\circ} 2$, maio-agosto de 2015; Boletim Epidemiológico, ano I, n 3, setembro-dezembro de 2015.

Figura 1 - Frequência de atendimentos no SOAMU/IEC por ano de ocorrência. Série histórica 2008-2015

Nos anos de 2012 e 2013, o SOAMU sinalizou a ocorrência de dois surtos de toxoplasmose, sendo um no Município de Ponta de Pedras e outro no Município de Barcarena. $O$ primeiro surto resultou na elaboração de um projeto de pesquisa que se encontra em execução no Laboratório de Toxoplasmose do IEC. Enquanto que, em 2014, ocorreu um surto de FT no bairro Castanheira, em plena região metropolitana de Belém; o SOAMU registrou 33 casos da doença ${ }^{5,6,7}$.

Outro exemplo recente da participação do SOAMU na vigilância foi registrado no mês de setembro do ano de 2015, quando a SESPA foi notificada da existência de casos suspeitos de FT entre três irmãos residentes no mesmo domicílio, no Município de Breves. Desses, um caso evoluiu com perfuração intestinal, a complicação mais temida dessa doença e a principal causa de óbito desses pacientes, pois o seu reparo é, invariavelmente, cirúrgico. Na semana posterior a essa ocorrência, houve registro de mais um caso suspeito da doença na mesma família. A partir do conhecimento desses casos, a SESPA buscou o apoio do Serviço de Epidemiologia do IEC, reunindo o Laboratório Central do Estado do 
Pará (LACEN/PA), o $8^{\circ}$ Centro Regional de Saúde e a Secretaria Municipal de Saúde de Breves. Foi realizada viagem de campo para a investigação do surto e aplicação das medidas de controle cabíveis. Nessa ação, a Seção de Bacteriologia e Micologia (SABMI) teve participação efetiva na confirmação diagnóstica em conjunto com o LACEN/PA.

Das análises laboratoriais processadas na SABMI/IEC, logrou-se obter 46 amostras positivas de 122 espécimes colhidos entre pacientes suspeitos e contatos intradomiciliares dos casos confirmados.

A FT está entre as principais causas de síndrome febril encaminhadas para o IEC em busca de definição diagnóstica, e o diagnóstico clínico dessa doença, muitas vezes, constitui um desafio em todos os níveis da atenção à saúde ${ }^{5,6}$. Nos últimos cinco anos, foram detectados 308 casos de FT no SOAMU, estabelecendo uma média de 61,6 casos anuais (Figura 2), refletindo a dura realidade dessa doença no âmbito do Estado do Pará, quiçá da Região Amazônica.

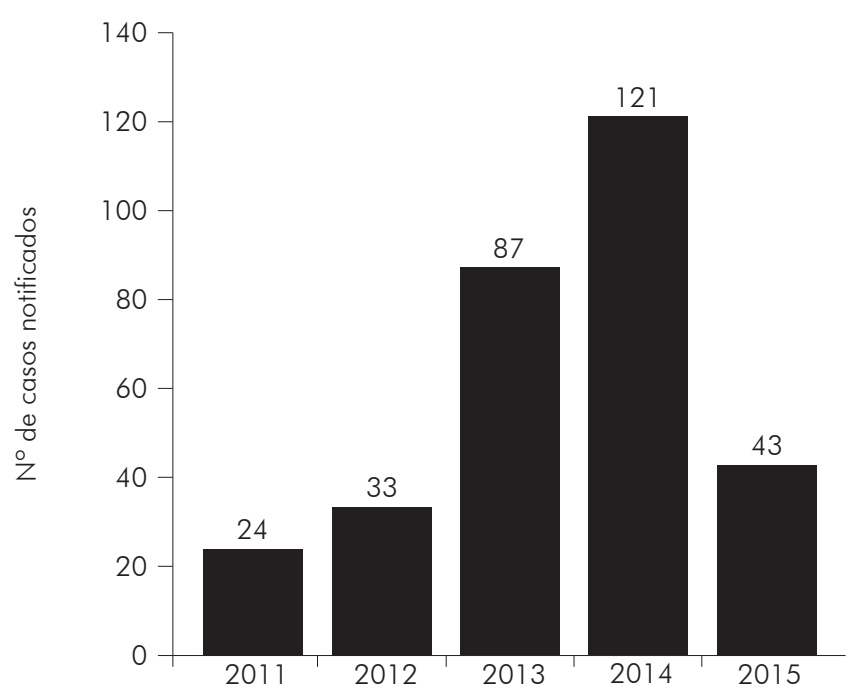

Fonte: Boletim Epidemiológico, ano I, $n^{\circ} 1$, janeiro-abril de 2015; Boletim Epidemiológico, ano I, $\mathrm{n}^{\circ} 2$, maio-agosto de 2015; Boletim Epidemiológico, ano l, $\mathrm{n}^{\circ} 3$, setembro-dezembro de 2015.

Figura 2 - Número de casos notificados de FT por ano de ocorrência. Série histórica, 2011-2015

As atividades de ensino, transversais à pesquisa e tão importantes quanto ela, também são realizadas no SOAMU. Como exemplo, destaca-se a atuação do setor em diversos níveis de ensino acadêmico ou técnico, tais como atividades de orientação oferecidas à graduação, incluindo programas de iniciação científica e internato eletivo; à especialização em doenças infecciosas, incluindo rodízios para residência médica da Universidade Federal do Pará e do Hospital Ophir Loyola; e ainda à pós-graduação em cursos de universidades públicas ou privadas com atividades presenciais, incluindo os Programas do IEC de Mestrado e Doutorado em Virologia e o recém-aprovado Mestrado em Epidemiologia e Vigilância em Saúde.

O Setor ainda promove a veiculação direcionada de informações e a comunicação imediata de resultados de pesquisa por meio da atividade de educação continuada idealizada para profissionais de nível técnico das Secretarias Municipais de Saúde, em parcerias assentadas em projetos de pesquisa; sendo esse um auxílio decisivo tanto para a execução das políticas de saúde elaboradas, quanto para a perspectiva de alterações das mesmas, em consonância com as mudanças da diversidade de cenários epidemiológicos que experimentamos continuamente.

\section{O IEC E O DESAFIO DAS DOENÇAS EMERGENTES: A PESQUISA DE POTENCIAL APLICAÇÃO NA ESTRUTURAÇÃO DAS POLÍTICAS DE SAÚDE}

Tendo o IEC iniciado suas atividades primordiais em investigações direcionadas para doenças parasitárias, à época, as desconhecidas e estranhas leishmanioses, o legado deixado pelos pioneiros às ulteriores gerações de pesquisadores, parece ter sido caracterizado, fundamentalmente, em dois quesitos: afinidade por desafios do conhecimento biomédico e o comprometimento com a saúde de populações negligenciadas. Assim, sucedeu-se com o desafio da DC na Amazônia, quando, no ano de 1969, pesquisadores locais chamaram atenção para uma estranha doença febril que acometeu simultaneamente quatro pessoas da mesma família no bairro de Canudos, em Belém, quando se aventara a possibilidade de uma transmissão inusitada e diferente daquela que ocorria em área endêmica clássica ${ }^{9}$.

Atualmente, a epidemiologia regional de DC está bem embasada em evidências observacionais e/ou analíticas sobre a transmissão veiculadora de grandes inóculos parasitários: a via oral, a qual permite expressão mórbida de doentes em fase aguda, sendo a síndrome febril sua principal manifestação e índices de letalidade por comprometimento miocárdico agudo, diretamente relacionado a diagnósticos tardios e a fatores imunológicos ou de comorbidades ainda pouco compreendidos ${ }^{10,11}$

Com o objetivo principal de seguir analisando a insólita epidemiologia regional e, simultaneamente, prestar assistência diagnóstica rápida e intervenção imediata em populações humanas expostas ao risco de adoecimento por DC, os estudos de cohorts dessas populações têm se mantido no IEC sob a forma de protocolos clínicos operacionais e estudos epidemiológicos em demandas ativas e passivas. Dentro do que propõe o Serviço de Epidemiologia, o SOAMU, em conjunto com o Laboratório de Doença de Chagas, soma, àquelas missões anteriormente identificadas, a responsabilidade de avaliar as pessoas afetadas ou expostas ao risco de adoecimento de forma contínua e sistemática.

Assim, os protocolos clínicos de pesquisa de seguimento de portadores de DC, iniciados em 2002, na Seção de Parasitologia (Laboratório de Doença de Chagas), e agora continuados no SOAMU, incluem uma sistemática melhor adaptada à atenção médica, identificando e acompanhando, seriadamente, cerca de 80 casos de DC em fase aguda (DCA) ou infectados, das mais diversas procedências no âmbito do Estado do Pará (Figura 3). 

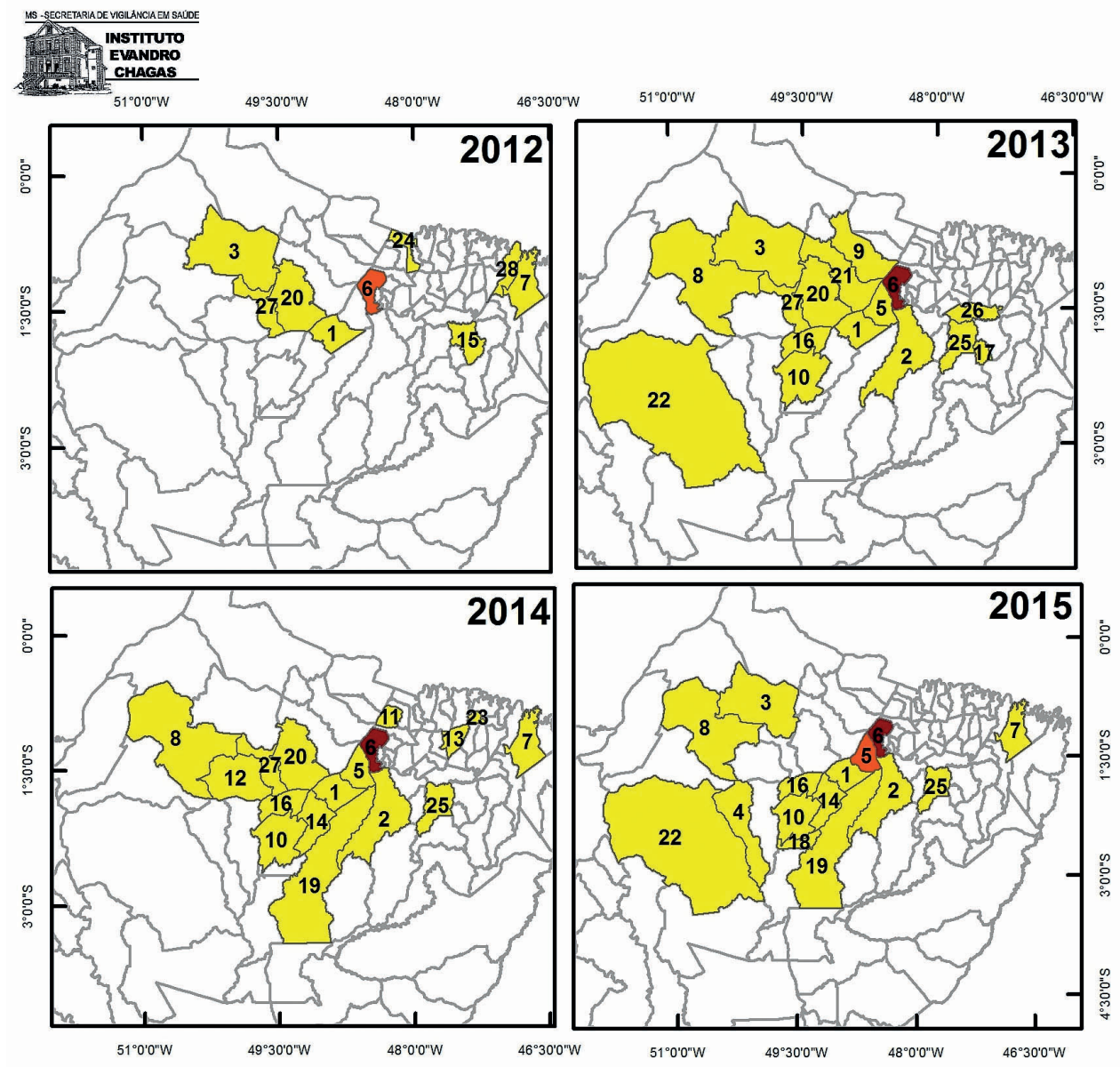

Processamento: 06/2016

Datum: SIRGAS 2000

Sistema de Coordenadas Geográficas

Dados:

BGE - Instituto Brasileiro de Geografia e Estatistica SOAMU/IEC/SVS/MS

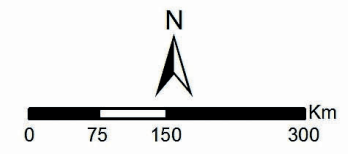

LABGEQ LABORATORIO DE GEOPROCESSAMENTO
DE DADDS EIDEMIOLOGICOS
INSTITUTO EVANDRO CHAGAS

\begin{tabular}{|r|c|}
\hline ID & MUNICIPIO \\
\hline 1 & ABAETETUBA \\
\hline 2 & ACARÁ \\
\hline 3 & ANAJÁS \\
\hline 4 & BAGRE \\
\hline 5 & BARCARENA \\
\hline 6 & BELÉM \\
\hline 7 & BRAGANÇA \\
\hline 8 & BREVES \\
\hline 9 & CACHOEIRA DO ARARI \\
\hline 10 & CAMETÁ \\
\hline 11 & COLARES \\
\hline 12 & CURRALINHO \\
\hline 13 & IGARAPÉ AÇU \\
\hline 14 & IGARAPÉ MIRI \\
\hline 15 & IRITUIA \\
\hline 16 & LIMOEIRO DO AJURU \\
\hline 17 & MÃE DO RIO \\
\hline 18 & MOCAJUBA \\
\hline 19 & MOJÚ \\
\hline 20 & MUANÁ \\
\hline 21 & PONTA DE PEDRAS \\
\hline 22 & PORTEL \\
\hline 23 & SANTARÉM NOVO \\
\hline 24 & SAO CAETANO DE ODIVELAS \\
\hline 25 & SAO DOMINGOS DO CAPIM \\
\hline 26 & SAO MIGUEL DO GUAMÁ \\
\hline 27 & SAO SEBASTIÃO DA BOA VISTA \\
\hline 28 & TRACUATEUA \\
\hline & \\
\hline & \\
\hline 19 & \\
\hline 19 & \\
\hline & \\
\hline & \\
\hline & \\
\hline
\end{tabular}

\section{Número de Casos de DCA}

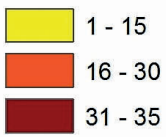

Figura 3 - Quantitativo de casos de doença de Chagas aguda e sua origem por municípios, identificados no IEC nos períodos entre 2012 e 2015

Pará é o Estado com maior número de casos, registrando $97,8 \%$ da autoctonia nos casos aqui atendidos. Comparativamente às taxas percentuais de casos de todo o Brasil, o Pará contribui com 74,7\% da autoctonia para o mesmo período ${ }^{12}$. A região metropolitana de Belém, que abrange os Municípios de Belém, Ananindeua, Marituba, Benevides, Santa Isabel do Pará, Santa Bárbara do Pará e Castanhal, detém 40 a 51,9\% da autoctonia nas incidências anuais de DC do Estado, seguida pelos Municípios de Abaetetuba e Barcarena, que pertencem à região do baixo rio Tocantins situada a nordeste do Pará.

O IEC tem identificado anualmente uma média de 15 surtos microepidêmicos de DCA (Figura 4). A partir dessa identificação, são desencadeadas e executadas investigações epidemiológicas em parceria com os setores de vigilâncias epidemiológicas municipais de saúde para a atenção diagnóstica rápida e intervenção terapêutica imediata.

Os surtos de DCA representam 70 a $80 \%$ da incidência anual e apresentam sazonalidade bem definida que se inicia nos meses de junho e julho (Figura 5), quando aumenta sua incidência, sendo a maioria deles considerada microssurto, por acometer frequentemente de duas a quatro pessoas simultaneamente.
Os surtos acontecidos em área urbana geram casos intrafamiliares e cumulativos anuais, por vezes repetindo-se em uma mesma localidade ou bairro da região metropolitana de Belém, em geral em bairros de populações carentes (Figura 6).

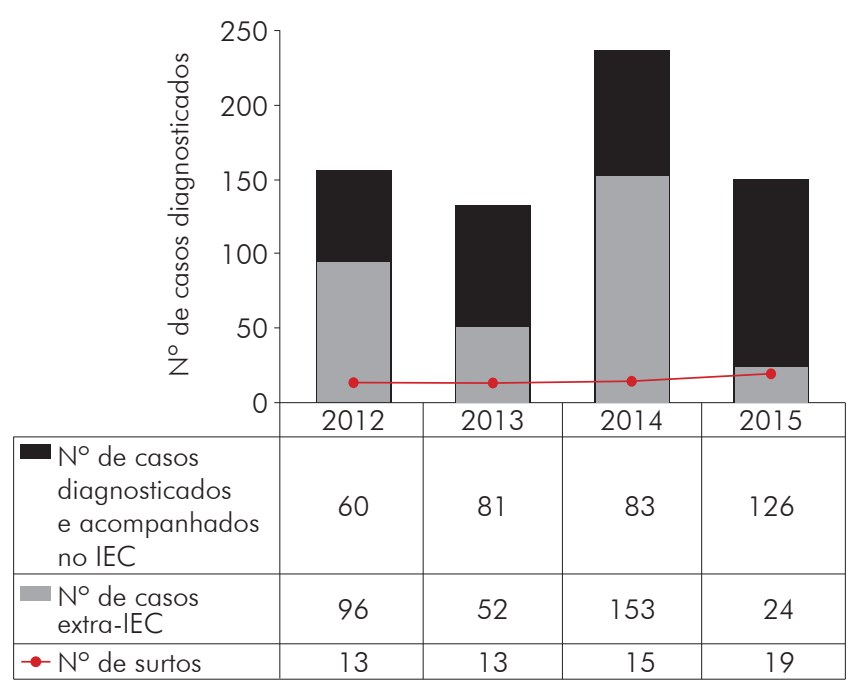

Fonte: IEC/SVS/MS e Gerência Técnica de Doença de Chagas, Pará/ SESPA.

Figura 4 - Série histórica de DCA no Brasil e número de surtos identificados no IEC, 2012-2015 


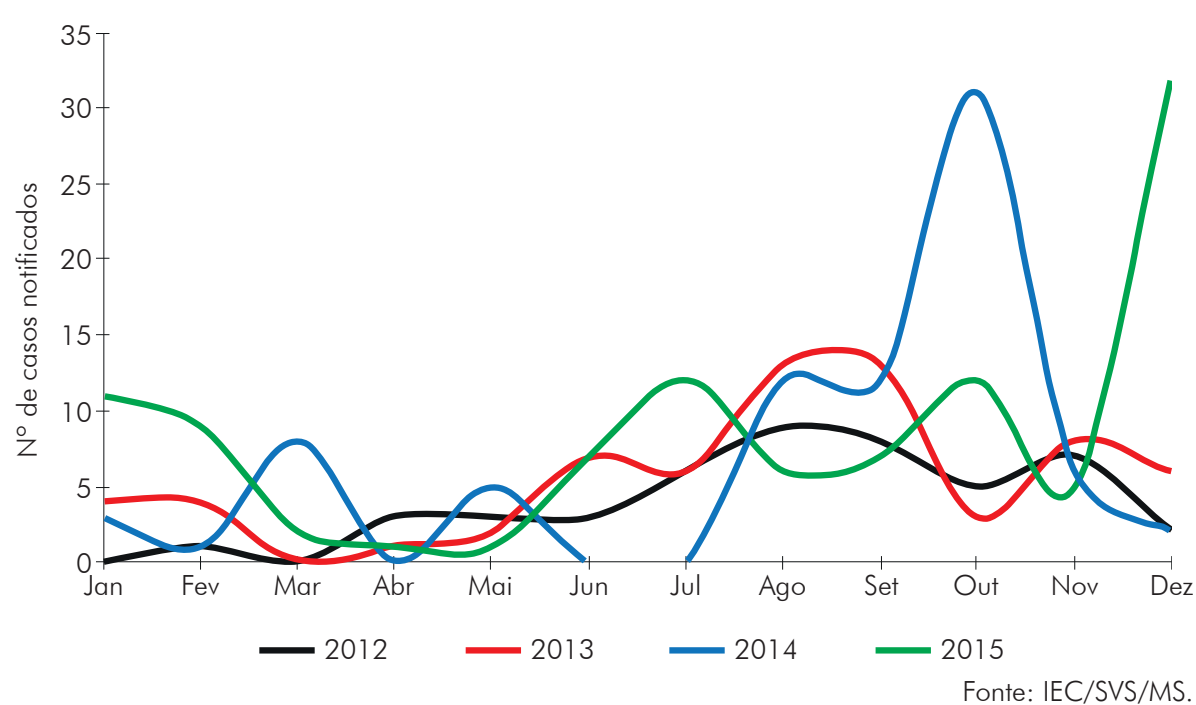

Figura 5 - Distribuição de casos de DCA diagnosticados no IEC por mês de adoecimento, 2012 a 2015

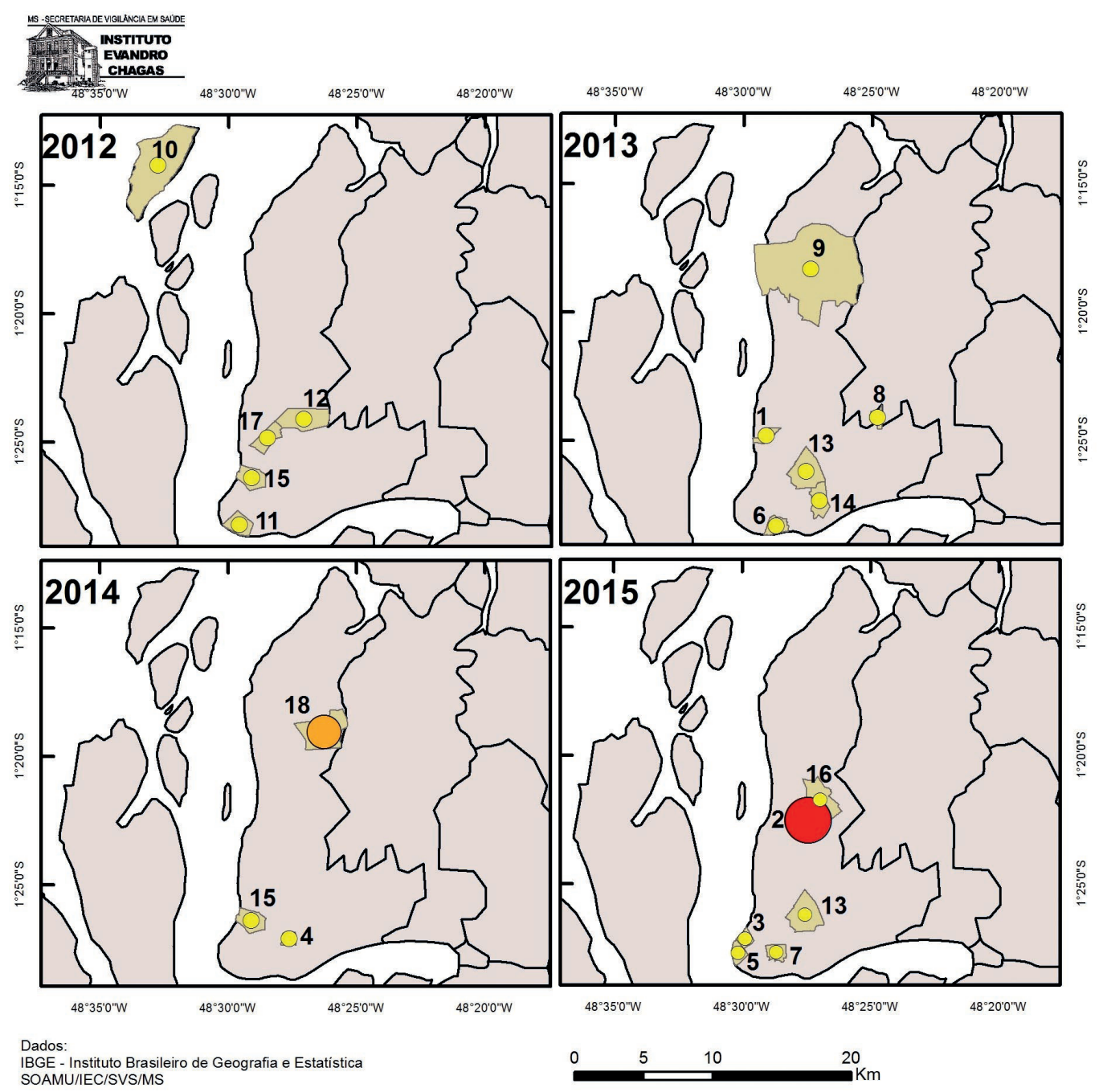

\section{LABGEQ}

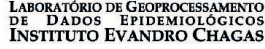

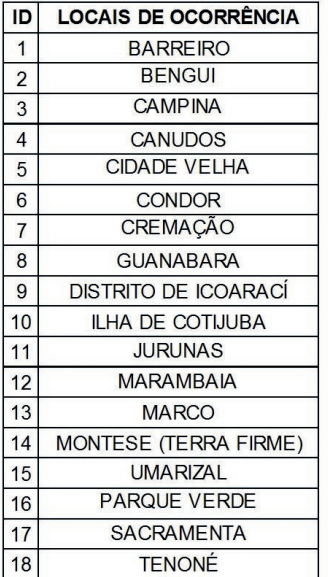

18 TENONÉ

$\bigwedge^{N}$

Número de Casos de DCA

○ 1-6

$7-14$

$15-26$

Processamento: 06/2016 Datum: SIRGAS 2000 Sistema de Coordenadas Geográficas

Figura 6 - Distribuição de surtos de DCA nos bairros de Belém e área insular periurbana, 2012 a 2015

Os estudos observacionais iniciados pelo grupo do IEC, desde 2002, e agora continuados, com a ênfase clínico-epidemiológica, propiciaram, portanto, a formulação de hipóteses sobre a inusitada transmissão?; comprovação da hipótese de transmissão por evidências observacionais e analíticas ${ }^{10,11,13}$; formulação de políticas de vigilância em saúde em auxílio decisivo à estrutura do sistema de vigilância epidemiológica de DC no Brasil ${ }^{14}$.

No que se refere à aplicação dos resultados dos estudos de cohort em DC na formulação de políticas de saúde, destaca-se: 
- Capacitação na estruturação das vigilâncias municipais dos Estados da Amazônia para - diagnóstico precoce de DC por meio da identificação rápida do $T$. cruzi, utilizando o método da gota espessa idealizada para microscopistas de malária, em parceria com os LACENs. Disso resultou o aumento imediato na sensibilidade da detecção de casos nos sistemas vigentes ${ }^{15}$.

- Indução à geração de conhecimentos no tema: proteção alimentar e fomento à vigilância rigorosa do controle sanitário alimentar ${ }^{14}$.

- Testagem contínua de esquemas alternativos de tratamento com as drogas usuais ou com novas drogas com base nas evidências da persistência de anticorpos anti-T. cruzi em indivíduos tratados em fase aguda (baixos níveis de cura sorológica) sem evidência de doença.

- Garantia presumida de atenção diagnóstica e intervenção medicamentosa e cardiológica imediata, de forma a prevenir evoluções graves ou potencialmente fatais, com base nas evidências do acometimento cardíaco em 56\% dos indivíduos com alterações de exames cardiológicos registrados em fase aguda. No mesmo sentido, a letalidade de 4,9\%, relacionada também ao acometimento cardíaco agudo, em especial à miopericardite, reforça a necessidade de tais garantias a serem proporcionadas pelos gestores em saúde ${ }^{16}$.

- Primeiras descrições de comportamento de vetores de T. cruzi em estudos de envolvimento populacional de comunidades ribeirinhas, com descrição de agressões intradomiciliares de triatomíneos a pessoas sem relação com doença evidente ${ }^{17}$.

\section{CONSIDERAÇÕES FINAIS}

A pesquisa translacional tem por objetivo promover pesquisa interdisciplinar e acelerar a troca bidirecional entre ciência básica e clínica para mover os achados de pesquisa básica do laboratório para ambientes aplicados, envolvendo pacientes e populações ${ }^{18,19}$.

Há muitos desafios ao conhecimento científico para a maioria das doenças infecciosas e seus agentes causais. É necessário e urgente que o conhecimento resultante das pesquisas básicas ganhe aplicabilidade prática na sociedade. No que se refere à prevenção, a indústria de vacinas tem sido a única a executar tal aplicabilidade, a partir de grandes investimentos, para os quais a ânsia pelo retorno financeiro, nos moldes que o mercado determina, dificulta a extensão dos seus benefícios a fronteiras onde estão populações carentes e desassistidas.

Uma avaliação aprofundada sobre a pesquisa translacional evidencia a fragilidade do tema quanto a sua abordagem prática. A pesquisa básica, cujos resultados possam ser efetivamente transmutados ou transportados para a aplicação à saúde de populações, tem permanecido no campo do discurso e da publicidade propulsora de mais investimentos que possam mantê-la. Anticorpos monoclonais e vacinas recombinantes prometem, por exemplo, a cura do câncer ou outras promessas anunciadas há mais de 20 anos e poucas delas têm sido eficazmente cumpridas $^{19}$. Soma-se a isso a expectativa social que exacerba as dúvidas sobre os resultados de tanto investimento em pesquisa básica e se isso atinge de forma ideal a translação em tempos compatíveis com as urgências. Vide o exemplo da epidemia de ebola no ano de 2015 que expôs a distância interposta entre as inovações científicas e tecnológicas e a falência da assistência médica urgente, diante da agilidade dos agentes infecciosos em suas adaptações contínuas de sobrevivência ${ }^{20}$.

serviço recém-criado no IEC experimenta a aplicabilidade rápida de resultados de pesquisa ao coadunar duas grandes habilidades institucionais: a de finalizar rapidamente diagnósticos difíceis de doenças que podem acontecer sob a forma de surtos; e a de controlar situações de emergência a elas relacionadas, leia-se, vigilância em saúde.

A complexidade de fatores determinantes de grande parte das doenças de notificação obrigatória, em especial aquelas que envolvem vetores biológicos e a segurança alimentar, é uma dificuldade especial enfrentada pelas estratégias de controle atualmente idealizadas. Paralelamente, as populações mais expostas são aquelas carentes do alcance de políticas públicas de atenção à saúde, como dever constitucional do Estado brasileiro, as chamadas populações negligenciadas. Configura-se, assim, a permanência de doenças seculares, hoje rotuladas de doenças negligenciadas e cujo entendimento merece investimento sustentado em pesquisa científica, se consideradas as constantes mudanças dos cenários epidemiológicos. Se não como pesquisa translacional na sua frágil concepção, mas minimamente cumprindo uma aplicação em vigilância de emergências.

Conclusivamente, a geração de demandas de pesquisa médica de alta qualidade, que auxilie no entendimento de agravos infecciosos de transmissão complexa e, simultaneamente, possa contribuir para as estratégias de controle do mesmo agravo e, ainda, assistir, com a mesma qualidade, populações carentes de atenção médico-diagnóstica, tem sido uma perspectiva fascinante ao longo de toda a trajetória institucional, hoje grandemente fortalecida com a criação do SOAMU, que revela, de maneira ampla, essas populações e doenças negligenciadas e propõe, a partir de seu potencial, o fortalecimento da pesquisa clínica.

\section{AGRADECIMENTOS}

À aguerrida equipe técnico-científica do SOAMU/SEVEP e à dra. Gilberta Bensabath, idealizadora pioneira do serviço. 


\section{REFERÊNCIAS}

1 Omran AR. The epidemiologic transition: a theory of the epidemiology of population change. Milbank Q. 1971 Oct;49(4 Pt 1):509-38.

2 Waldman EA, Silva LJ, Monteiro CA. Trajetória das doenças infecciosas: da eliminação da poliomielite à reintrodução da cólera. Inf Epidemiol Sus. 1999 set;8(3):5-47.

3 Carmo EH, Barreto ML, Silva Jr JB. Mudanças nos padrões de morbimortalidade da população brasileira: os desafios para um novo século. Epidemiol Serv Saude. 2003 jun;12(2): 63-75.

4 Brasil. Ministério da Saúde. Portaria $n^{\circ} 204$, de 17 de fevereiro de 2016. Define a Lista Nacional de Notificação Compulsória de doenças, agravos e eventos de saúde pública nos serviços de saúde públicos e privados em todo o território nacional, nos termos do anexo e dá outras providências. Diário Oficial da União, Brasília (DF), 2016 fev 18; Seção 1:23.

5 Ministério da Saúde (BR). Secretaria de Vigilância em Saúde. Instituto Evandro Chagas. Bol Epidemiol Inst Evandro Chagas. 2015 jan-abr;1 (1):1-4.

6 Ministério da Saúde (BR). Secretaria de Vigilância em Saúde. Instituto Evandro Chagas. Bol Epidemiol Inst Evandro Chagas. 2015 mai-ago;1 (2):1-4.

7 Ministério da Saúde (BR). Secretaria de Vigilância em Saúde. Instituto Evandro Chagas. Bol Epidemiol Inst Evandro Chagas. 2015 set-dez; 1 (3):1-6.

8 Ministério da Saúde (BR). Fundação de Serviços de Saúde Pública. Instituto Evandro Chagas: 50 anos de contribuição às ciências biológicas e à Medicina Tropical. Vol. 1. Belém: FSESP; 1986.

9 Shaw J, Lainson R, Fraiha H. Considerações sobre a epidemiologia dos primeiros casos autóctones de doença de Chagas registrados em Belém, Pará, Brasil. Rev Saude Publica. 1969 dez;3(2): 153-7.

10 Valente SAS, Valente VC, Pinto AYN, César MJB, Santos MP, Miranda COS, et al. Analysis of an acute Chagas disease outbreak in the Brazilian Amazon: human cases, triatomines, reservoir mammals and parasites. Trans R Soc Trop Med Hyg. 2009 Mar;103(3):291-7.

11 Pinto AYN, Valente SA, Valente VC, Ferreira Junior AG, Coura JR. Fase aguda da doença de Chagas na Amazônia brasileira. Estudo de 233 casos do Pará, Amapá e Maranhão observados entre 1988 e 2005. Rev Soc Bras Med Trop. 2008 nov-dez;41(6):602-14.
12 Ministério da Saúde (BR). Secretaria de Vigilância em Saúde. Doença de Chagas aguda no Brasil: série histórica de 2000 a 2013. Bol Epidemiol. 2015;46(21):1-9.

13 Beltrão HBM, Cerroni MP, Freitas DRC, Pinto AYN, Valente VC, Valente SA, et al. Investigation of two outbreaks of suspected oral transmission of acute Chagas disease in the Amazon region, Pará State, Brazil, in 2007. Trop Doct. 2009 Oct;39(4):231-2.

14 Organização Pan-Americana da Saúde. Área de vigilância sanitária e manejo de doenças. Guia para vigilância, prevenção, controle e manejo clínico da doença de Chagas aguda transmitida por alimentos. Brasília: OPAS; 2009. 92 p. (Série de manuais técnicos; 12).

15 Pinto AYN, Santiago A, Miranda K, Valente SAS, Souza JM, Oliveira SM, et al. Detecção precoce de casos de doença de Chagas no Pará: resultados parciais de eficiência da estratégia de busca diagnóstica de T. cruzi em um posto notificador de malária em Belém. In: Resumos do $45^{\circ}$ Congresso da Sociedade Brasileira de Medicina Tropical; 2009 mar 3-7; Recife, Brasil. Uberaba: Sociedade Brasileira de Medicina Tropical; 2009. p. 525.

16 Pinto AYN, Ferreira SMAG, Valente SAS, Valente VC, Ferreira Jr AG. Alterações eletrocardiográficas durante e após tratamento com benzonidazol em fase aguda de doença de Chagas autóctone da Amazônia brasileira. Rev Pan-Amaz Saude. 2010 jun;1 (2):67-76.

17 Pinto AYN, Santos JE, Maciel RF, Fernandes AS, Guerreiro JF, Valente VC, et al. Dupla abordagem de infecções por Trypanosoma cruzi e/ou Plasmodium spp. aplicada ao diagnóstico de doença de Chagas e exposição vetorial triatomínica na Amazônia brasileira. Rev Pan-Amaz Saude. 2015 mar;6(1):35-43.

18 National Cancer Institute. National Cancer Advisory Board. Advancing translational cancer research: a vision of the Cancer Center and SPORE Programs of the future [Internet]. Bethesda: NCl; 2003 [cited 2013 Jan 12]. Available from: http://deainfo.nci. nih.gov/advisory/ncab/workgroup/p30-p50/P30P50final 12 feb03.pdf.

19 Guimarães R. Pesquisa Translacional: uma interpretação. Cienc Saude Coletiva. 2013 jun; 18(6):1731-44.

20 Bell BP, Damon IK, Jernigan DB, Kenyon TA, Nichol ST, O'Connor JP, et al. Overview, control strategies, and lessons learned in the CDC response to the 2014-2016 Ebola epidemic. MMWR Suppl. 2016 Jul;65(3):4-1 1 . 